\title{
Nanowires in Thermoelectric Devices
}

\author{
Keivan Davami and Jeong-Soo Lee \\ Division of IT Convergence Engineering, Pohang University of Science and Technology, Pohang 790-784, Korea
}

\author{
M. Meyyappan ${ }^{\dagger}$ \\ Division of IT Convergence Engineering, Pohang University of Science and Technology, Pohang 790-784, Korea and \\ National Aeronautics and Space Administration Ames Research Center, Moffett Field, CA, USA
}

\begin{abstract}
The low efficiency of bulk thermoelectric materials has limited the widespread application of thermoelectric power generation. Theoretical and experimental investigations indicate that materials prepared in the form of nanowires show higher thermoelectric coefficients, thus promising to revolutionize the field. This article reviews the basics of thermoelectric power generation, conventional devices, the role of nanowires and the current status of the field.
\end{abstract}

Keywords: Thermoelectric, Nanowires, Seebeck coefficient, Waste heat recovery

\section{INTRODUCTION}

The dependence on fossil fuels for most of our energy needs has become a serious issue due to the adverse effect on global economy brought by the increasing costs and the volatility of the fuel market. The negative impact on global warming is a long term issue pushing attention to alternative, cleaner, and cheaper energy sources. In this regard, harvesting solar and wind energy sources is a major focus throughout the world, and to a lesser extent, recapturing waste heat as power offers a solution to many our energy problems. In many scenarios in daily life, heat is just dissipated without deriving any benefit, for example, the heat produced by the car engine. Thermoelectric materials have made it possible to convert that thermal energy into electrical energy [1-5]. The reversible process of refrigeration is also possible by supplying an electrical current to produce a thermal gradient (Fig. 1). Thermoelectric devices do not have moving parts, create pollution, or make noise, thus offering an attractive source of energy.

A thermoelectric device is a combination of $p-n$ junctions

${ }^{\dagger}$ Author to whom all correspondence should be addressed: E-mail:m.meyyappan@nasa.gov

Copyright $@ 2011$ KIEEME. All rights reserved. This is an open-access article distributed under the terms of the Creative Commons Attribution Non-Commercial
License (http:///creativecommons.org/licenses/by-nc/3.0) which permits unrestricted noncommercial use, distribution, and reproduction in any medium, provided the original work is properly cited. which are connected electrically and thermally in series and parallel respectively. Many such $\mathrm{p}-\mathrm{n}$ junctions are needed to generate the desirable output voltage since each p-n junction in a system only generates a small voltage difference. Bulk thermoelectric materials do not have high efficiency, limiting wide applications at present. The figure of merit for a thermoelectric material, ZT, is given as:

$$
Z T=S^{2} \sigma T / \kappa
$$

where $\mathrm{S}$ is the Seebeck coefficient, $\sigma$ is the electrical conductivity, $\mathrm{T}$ is the temperature and $\kappa$ is the thermal conductivity. The product $S^{2} \sigma$ is also known as power factor. There has been a tremendous amount of research in the last decade to improve the ZT beyond that of bulk materials. Nanomaterials appear to provide an avenue to achieve this goal, for example, making nanocomposites, adding nanoparticles to a bulk material, or employing one-dimensional nanostructures. Significant amount of research has been reported [6-11] on nano/micro-grained composites and adding nanopaticles to a bulk thermoelectric material. However, this review does not cover these developments but focuses only on the use of nanowires in thermoelectric power generation. A background on the physics of thermoelectricity is given first, followed by conventional devices. The rationale for using nanowires in this endeavor and the current status are discussed next and finally, a summary of challenges and future 
(a)

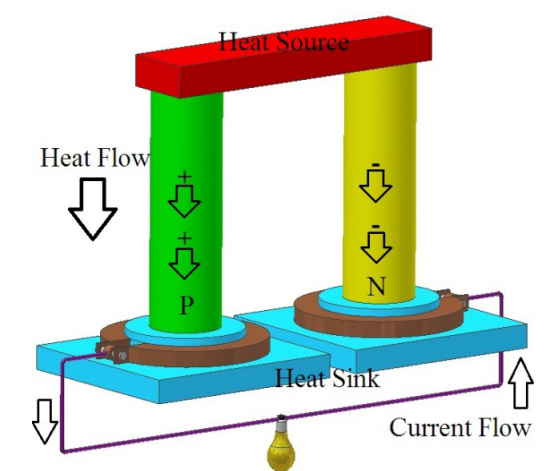

(b)

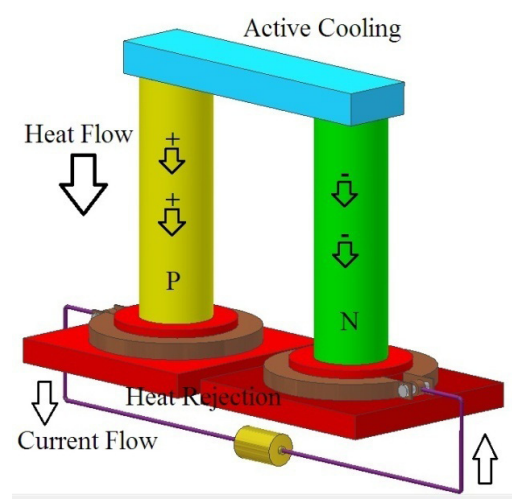

Fig. 1. A schematic of a thermoelectric module (a) power generation, (b) active refrigeration. In (a), an applied temperature difference causes electrons or holes to diffuse from the hot side to the cold side, resulting in current flow through the circuit and in (b), heat evolves at the upper junction and is absorbed at the lower junction when a current is made to flow through the circuit.

outlook is presented.

\section{BACKGROUND ON THERMOELECTRICITY}

In 1821, Thomas Johann Seebeck discovered the thermoelectric effect while doing a simple experiment using a circuit made from two different metals, with the junctions maintained at different temperatures. He noticed that this would deflect a compass magnet and later realized that a "thermoelectric force" induced an electrical current, which according to Ampere's law deflects the magnet. Thermoelectricity is attributed to the thermal diffusion of charge carriers in a material. Hot charge carriers in a thermoelectric material diffuse to the cold end of the material much more than the cold charge carriers diffusing to the hot end, and this proceeds until a state of dynamic equilibrium is reached. This phenomenon is known as the Seebeck effect. The voltage is proportional to the temperature difference between the two junctions. This relation between the temperature difference and the output voltage defines an important parameter called the "Seebeck coefficient", or thermopower. Themoelectric power (TEP), $S$ (units: $\mu \mathrm{V} / \mathrm{K}$ ) is one of the basic characteristics of a material; similar to other properties of a material such as electrical conductivity, it can be measured and classified for a material. The thermoelectric voltage can be either negative or positive depending on if the holes or electrons are charge carriers. The Seebeck coefficient is given by:

$$
S=\frac{\Delta V}{\Delta T}
$$

Several years after Seebeck's experiments, a French physicist in 1834 discovered that a current passing through a junction of two dissimilar metals produced heating or cooling. It was possible to freeze water into ice or melt a cube of ice, depending on the direction of the current. The heat created (or absorbed), q, is proportional to the current, I and the coefficient relating the two is called the Peltier coefficient.

$$
\pi=q / I
$$

The Seebeck coefficient and electrical conductivity depend only on the electronic properties of the material whereas the thermal conductivity depends on contributions from both the electronic component and lattice vibrations. Achieving high efficiency requires a high $S$ to realize a large voltage for a small temperature difference, a high $\sigma$ to minimize Joule heating losses, and a small $\kappa$ to maintain a high temperature difference. In reality, it has been very challenging to reach a high ZT (for example ZT >1) since the parameters mentioned above are not independent of each other, and any attempt to improve one might adversely affect the other. For example, an increase in electrical conductivity invariably accompanies an increase in thermal conductivity. A thermoelectric device with a ZT higher than 3 is necessary to be competitive with conventional refrigeration or power generation.

\section{BULK AND THIN FILM DEVICES}

Semiconductor materials exhibit high Seebeck coefficients which makes them attractive as efficient heat pumps. While the best metal has a Seebeck coefficient of $\sim 30 \mu \mathrm{V} / \mathrm{K}$, some semiconductors have Seebeck coefficients in the range of $100-200 \mu \mathrm{V} / \mathrm{K}$. In metals, only a small fraction of electrons at high energies are capable of changing energy states and exchanging energy with the surrounding environment according to the Pauli Exclusion Principle. This fraction can be predicted using Fermi-Dirac statistics:

$$
C_{e l-m}=\frac{\pi^{2} \kappa_{B} T}{2 \varsigma_{0}}
$$

where $\zeta_{0}$ is the Fermi energy [2]. For semiconductors, because of the energy gap, Maxwell-Boltzmann statistics can be used to approximate the number of electrons contributing to an electronic specific heat.

$$
C_{e l-s}=\kappa_{B}\left(\frac{3}{2}-\frac{U}{\kappa_{B} T}\right)
$$

where $U$ is the excitation energy per electron [2]. The second term in the above equation implies that the number of charge carriers in the conduction band will go up when the temperature increases. The electrons in the relatively empty conduction band in semiconductors are more capable of changing energy states than in the case of metals which causes an increase in the electronic specific heat.

SiGe, $\mathrm{PbTe}$ and $\mathrm{Bi}_{2} \mathrm{Te}_{3}$ alloys are some of the popular semiconductor materials considered for thermoelectric applications. In 
principle, there is no theoretical limit for the efficiency of a thermoelectric device, however, practical considerations such as the limitation in the ratio of $\sigma / \kappa_{\mathrm{e}}$ (where $\kappa_{\mathrm{e}}$ is the electronic contribution to the thermal conductivity according to the Weidmann-Franz law) make it hard to improve the efficiency. Consequently, the largest ZT value realized to date in applications is around 1 for $\mathrm{Bi}_{2} \mathrm{Te}_{3}$.

A bulk semiconductor with a high atomic weight and a reasonable $\mathrm{S}$ and $\sigma$ is expected to have a low thermal conductivity, resulting in a large $\mathrm{ZT}$. This expectation is due to the reduced speed of sound in such a material, but this has not been realized yet [12]. New bulk materials with higher ZT have been under investigation in recent years. Rhyee et al. [13] reported a ZT of $\sim 1.5$ at $705 \mathrm{~K}$ in a binary crystalline bulk n-type $\operatorname{In}_{4} \mathrm{~S}_{3-\delta}$ attributing the high value to the high Seebeck coefficient and the low thermal conductivity in the plane of the charge density wave.

The efficiency of a thermoelectric element is related to ZT as follows [14]:

$$
\eta_{\max }=\frac{\Delta T}{T_{H}} \frac{\sqrt{1+Z T}-1}{\sqrt{1+Z T}+\frac{T_{C}}{T_{H}}}
$$

where $T_{H}$ and $T_{C}$ are the temperatures of the hot and cold junctions respectively, and $\frac{\Delta T}{T_{H}}$ is the Carnot efficiency, which places a fundamental limit on the efficiency of all heat engines. The efficiency of a thermoelectric device would reach the Carnot limit only with an infinite ZT according to this relation.

\section{CURRENT THERMOELECTRIC APPLICATIONS}

Currently, a variety of thermocouples, Petlier coolers and even automobile seat warming/cooling systems made of thermoelectric materials are available in the market. Thermoelectric generators have been used in satellites and space probes. For example, in a "radioisotope thermoelectric generator" (RTG), radioactive decay releases heat and an array of thermocouples convert this heat into electricity through the Seebeck effect. Plutonium-238 as oxide Pu02 is used in this system which emits alpha particles during the isotopic decay process and the emitted particles collide with the walls of the containers, creating the heat for the thermoelectric converter [15]. Such devices have been developed and implemented by NASA in various space missions including Apollo, Pioneer, Viking, Voyager, Galileo, and Cassini.

For the automotive sector, Amerigon Inc. designed and fabricated a climate control seat system used in the front seat on two models of the Jaguar XF mid-size luxury sports sedans, as well as the Nissan Maxima. This system allows the driver to warm or cool the seats as needed seasonally [16]. Other commercialized devices include water coolers, cool/heat sleep pads, and personal mini coolers [17]. A Japanese company recently developed [18] a device for charging cell phones, lap tops, etc., with the purpose to help people who have lost their homes due to the 2011 tsunami in Japan. A pan in this device contains a thermoelectric material at the bottom with one end in direct contact with the fire while the other end has an interface with the boiling water in the pan. The average temperature of the fire is $\sim 600^{\circ} \mathrm{C}$ and with water boiling at $100{ }^{\circ} \mathrm{C}$, the difference in temperature can be used to produce electricity.

\section{NANOMATERIALS FOR THERMOELECTRIC APPLICATIONS}

Nanomaterials have renewed the interest in thermoelectricity by allowing to tailor the interdependent parameters, thus providing new avenues to increase the efficiency of thermoelectric devices $[19,20]$. Low thermal conductivity and a wide range of density of states and phonon electron scattering in nanomaterials make them ideal for thermoelectric applications [21,22]. When the dimensions of a material are about the mean free path of electrons, the density of states increases due to quantum confinement [23], which causes an increase in the Seebeck coefficient [24]. Moreover, the thermal conductivity of the material decreases due to the surface scattering mechanism, since the dimensions are less than the mean free path of phonons.

Alloying used to be the main approach to achieve increased efficiency. Since the 1990s, two different alternatives have become popular: the first one involving new materials with complex crystalline structures and the second by reducing the size of the candidate material [25]. Simple alloying was replaced by nano inclusions when the field of nanotechnology started to receive attention, following theoretical predictions that nano-inclusion reduces the thermal conductivity more than alloying [26]. Tuning of the transport parameters has been shown to be possible in the case of microstructural modifications in nano/micro composites. When the grain size increases, the carrier mobility increases resulting in an increase in electrical conductivity. It is also practical to reduce the thermal conductivity by increasing the grain boundary scattering [25].

Dresselhaus et al. [23] first reported the possibility of increasing the power factor by taking advantage of quantum confinement effects. Since then, research on different low-dimensional systems including superlattices, nanowires, and quantum dots has intensified. Venkatasubramanian et al. reported a ZT of 2.4 for $\mathrm{Bi}_{2} \mathrm{Te}_{3} / \mathrm{Sb}_{2} \mathrm{Te}_{3}$ [27] by controlling the transport of phonons and electrons in the superlattices; a temperature drop of $32 \mathrm{~K}$ was shown possible at room temperature in a device using this material. Harman et al. obtained a ZT larger than 3.0 at $600 \mathrm{~K}$ for $\mathrm{PbTe} / \mathrm{PbTeSe}$ quantum dot superlattices [28].

\section{NANOWIRE-BASED DEVELOPMENTS}

The single crystalline nature of nanowires is more advantageous than superlattice films since the electrical conductivity in nanowires is not limited by charge scattering, tunneling, and hopping [29]. In addition, electrical conductivity can be optimized by doping as well. Surface scattering in nanowires [30] changes the lattice component of thermal conductivity $\left(\kappa_{l}\right)$ in the thermoelectric figure of merit:

$$
Z T=\frac{\sigma S^{2} T}{\kappa_{e}+\kappa_{l}}
$$

While size reduction in nanowires confines the movement of the electrons to one direction, it does not restrict the motion of phonons. The motion restriction of electrons improves the electrical conductivity and thus, modifies Seebeck coefficient as desired [30,31]. However, the electronic component of thermal conductivity also increases too, which is undesirable. The lattice component of thermal conductivity can be estimated from:

$$
\kappa_{L}=\frac{1}{3} C_{g} v_{S} l
$$

where $v_{S}$ is the speed of sound in the material and $l$ is the total mean free path. If the diameter of the nanowire is smaller than the mean free path in the above equation, $l$ is replaced with the 
(a)

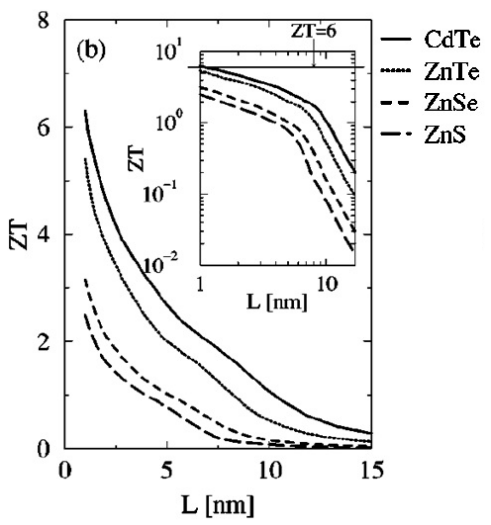

(b)

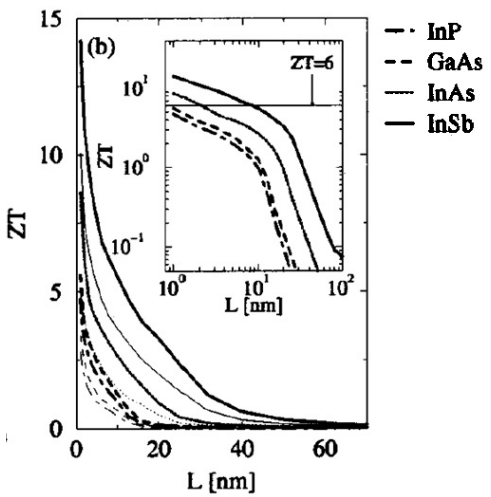

Fig. 2. Maximum room temperature ZT vs. nanowire diameter. Inset: same plot in logarithmic scale. (a) group II-VI and (b) group III- V. Reproduced with permission from [33,34].

diameter of the nanowire; then, the thermal conductivity will reduce with the size of the nanowire.

Nanowires have been predicted to show interesting properties for diameters less than $100 \mathrm{~nm}$ [32]. Mingo calculated the ZT for various II-VI and III-V semiconductors in bulk and nanowire formss $[33,34]$ using an iterative solution for the Boltzmann transport to model the electronic transport and a full transmission function approach for the prediction of the lattice thermal conductivity. His results showed that (i) ZT increases with a decrease in nanowire diameter, (ii) CdTe in II-VI semiconductors and InSb in III-V semiconductors are the best choices for thermoelectric applications, and (iii) II-VI nanowires have smaller ZTs compared to III-V materials for a given nanowire diameter. Based on his predictions reproduced in Figure 2, nanowires with diameters smaller than $\sim 5 \mathrm{~nm}$ are needed in order to obtain ZT greater than 3 .

Size effects on thermal conductivity and ZT of nanowires have been investigated extensively. A theoretical study [35] based on the Boltzmann transport equation and ab initio electronic structure calculations show possibilities to obtain ZT values greater than 1 for very thin nanowires $(\mathrm{d}<3 \mathrm{~nm})$ if the ionic thermal conductivity is reduced by about 2 or 3 orders of magnitudes. Boukai et al. [36] showed that while bulk silicon is a weak thermoelectric material mostly because of its low thermal conductivity, Si nanowires have the potential to yield higher efficiency. They showed that a ZT of $\sim 1$ is possible for Si nanowires by optimizing the nanowire size and tuning the doping level at $200 \mathrm{~K}$ due to the reduction in thermal conductivity. The nanowire size effects on the thermal conductivity and electrical conductivity of $\mathrm{PbTe}$ nanowires have been reported [37] showing the possibility to increase the Seebeck coefficient up to $-130 \mu \mathrm{V} / \mathrm{K}$ and reduce the thermal conductivity to $1.2 \mathrm{~W} / \mathrm{m} . \mathrm{K}$ (Fig. 3). Thermal conduc- (a)

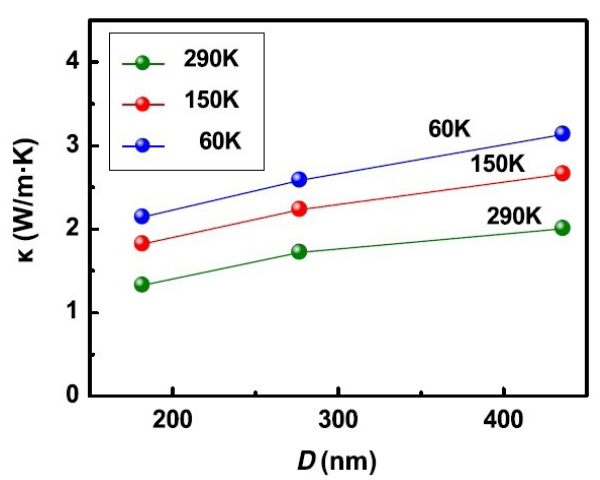

(b)

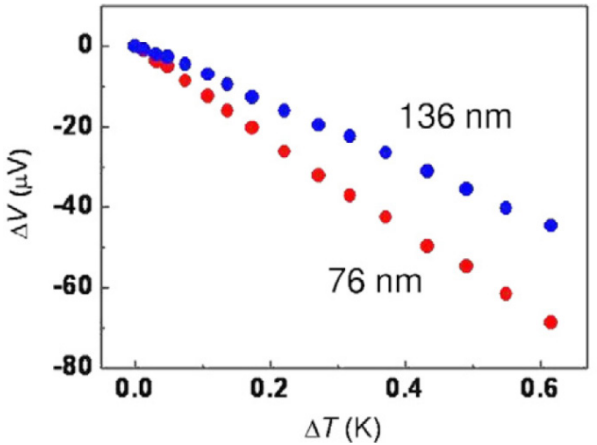

Fig. 3. Diameter-dependent thermal conductivity of individual $\mathrm{PbTe}$ nanowires. The temperatures are 60, 150 and $290 \mathrm{~K}$, respectively. TEPs (S) of -130 and $-76 \mu V \mathrm{~K}^{-1}$ are obtained from the slope of a linear fit to the data at $300 \mathrm{~K}$. Reproduced with permission from [37].

tivity of PbSe nanowires was found to be lower than bulk PbSe by a factor of 10-1000 at various temperatures [38].

The potential for obtaining high ZT using heterostructures and hollow nanostructures has been explored by many groups $[39,40]$. Nano-heterostructures may exhibit a low thermal conductivity due to interface phonon scattering [41]. A core-shell structure is another effective method for reducing the thermal conductivity where the rough interface acts as a secondary scattering phase. Kang et al. [41] reported the possibility to reduce the thermal conductivity of $\mathrm{Bi} / \mathrm{Te}$ core-shell nanowires to an amount close to the amorphous limit. While the electrical conductivity of the core/shell nanowires does not change appreciably, their thermal conductivity can be five times lower than the conventional nanowire of the same size. These findings have also been validated by molecular dynamic simulations [42]. The degradation of thermal conductivity of $\mathrm{Si} / \mathrm{Ge}$ core/shell nanowires is attributed to the depression and localization of long-wavelength phonon modes at the interface of $\mathrm{Si} / \mathrm{Ge}$ as well as high frequency non-propagating diffusive modes.

Hochbaum et al. [43] reported that rough Si nanowires have a low thermal conductivity around $1.6 \mathrm{Wm}^{-1} \mathrm{~K}^{-1}$, attributing it to the higher rates of diffuse reflection or backscattering of phonons at the interfaces. They also showed that Si nanowires smaller than $50 \mathrm{~nm}$ in diameter exhibit two orders of magnitude reduction in thermal conductivity. Several experimental and theoretical studies have focused on the effects of growth direction of the nanowires on thermoelectric properties $[35,44]$. The growth direction affects the charge and energy transport in nanowires. For example, anisotropy in thermal conductivity of single-crystal Bi nanowires has been reported [44], with [110] nanowires showing a thermal conductivity about a quarter of that of [ $\overline{1} 02]$ nanowires. Computational studies [35] confirm that electrical conductivity, Seebeck coefficient, and electron thermal conductivity of silicon nanowires are strongly dependent on the growth direction 


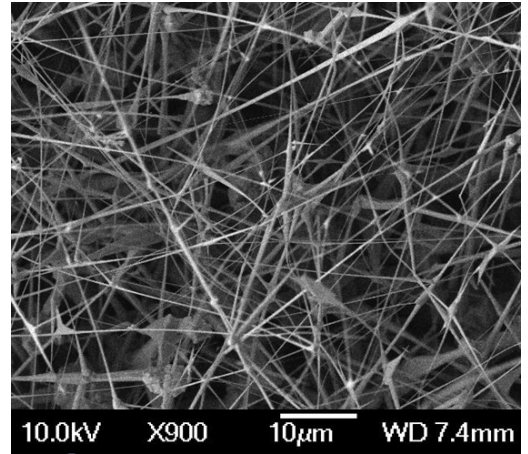

Fig. 4. A mat of ZnTe nanowires grown by the vapor-liquid-solid (VLS) technique.

of the nanowire. Si nanowires with a $1 \mathrm{~nm}$ diameter grown in [011] have the highest values of $\sigma$ and $\kappa_{\mathrm{e}}$ for a given carrier concentration. For nanowires with a diameter of $3 \mathrm{~nm}$, wires with a growth direction of [011] show the highest electrical conductivity. These studies indicate the need to consider growth direction in the design of future devices since it can affect the efficiency considerably.

Various methods have been used for the growth of thermoelectric nanowires including sol-gel techniques, template synthesis and vapor-liquid-solid (VLS) approach using catalysts [46]. Typically, chemical synthesis techniques performed at low temperatures do not yield high quality nanowires suitable for electronics applications as these structures tend to have many defects. Template-based techniques, while allowing to grow vertical nanowires confined into the pores, template removal and possible resultant damages pose an issue in device fabrication. Compared to these two techniques, the VLS approach is more popular since it is similar to chemical vapor deposition except for the use of a catalyst to facilitate nanowire formation [4648]. Figure 4 shows a mat of ZnTe nanowires grown using VLS method. Regardless of the approach, precise diameter control of the nanowires is currently elusive. Precise positioning of the nanowires with controlled diameters has been attempted using nanoporous templates [49], scanning probe lithography [50], AFM resist scratching and liftoff [51], and nano polymer wires or tubes as templates [52]. Zhou et al. [53] reported that the growth pressure affects the thermoelectric properties of InSb nanowires synthesized by the VLS method. The Seebeck coefficient of InSb nanowires is found to be smaller than bulk InSb and nanowires grown at lower pressure have a higher Seebeck coefficient. This observation was attributed to the formation of Sb-doped InSb NWs due to the loss of In, since In is easily oxidized by the remaining oxygen in the growth tube. Their samples also had a thin layer of indium oxide and its effect on the observations made is not clear.

The measured ZT values of nanowires in many cases appear to be lower than theoretical predictions and the main reason may be due to the low measured electrical conductivity of the nanowires. The charge carrier mobility of the nanowires is very low due to the surface trap states and point defects. Annealing can possibly reduce the defect density [54] and subsequently increase the electrical conductivity. Annealing of p-type PbSe [29] was shown to decrease the resistivity of the nanowires significantly, with the power factor value of ZT reaching $0.04 \mathrm{ohm} . \mathrm{cm}$. The approaches commonly used for making these measurements is discussed next.

Most experimental studies focused on a mat of nanowires for measurement of thermoelectric properties due to the difficulties associated with isolating and positioning a single nanowire [55].

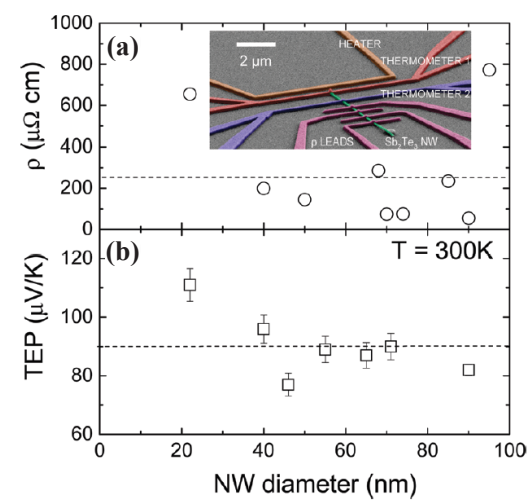

Fig. 5. (a) Resistivity and (b) thermopower as a function of NW diameter at $300 \mathrm{~K}$. Inset shows a color-enhanced SEM image of a typical device. The dashed lines in (a) and (b) are bulk values. Reproduced with permission from [59].

It is not possible then to evaluate the properties as a function of size, impurities, etc. In order to measure the thermoelectric properties of a single nanowire, a test device small enough to make the measurement is needed and two methods have been commonly used in the literature. In the first method, a thick layer of silicon dioxide is formed on the silicon substrate. The low thermal conductivity of $\mathrm{SiO}_{2}$ helps to obtain the required temperature difference between the two ends of the nanowire. Then, several drops of the nanowire dispersion are placed on the substrate and after the solvent evaporates in a few minutes, the nanowires remain on the substrate. Subsequently, gold (or Pt) electrical contacts are fabricated using photolithography and electron beam evaporation. One of the electrodes acts as a heater, and Joule heating through a heater line is used to establish a temperature difference which generates a thermoelectric voltage in the nanowire [56-58]. The Seebeck coefficient of the nanowire is calculated using $\Delta \mathrm{T}$ and measuring the Seebeck voltage. Though this approach is simple and provides good electrical contacts, it is difficult to establish a large temperature difference between the two ends of the nanowire since the nanowire is on a huge thermal mass of silicon oxide (Fig. 5). Thermoelectric properties of individual single crystal antimony telluride nanowires of varying diameters were measured using such a device [59] which showed that the resistivity of the nanowires is close to the bulk value. An increase of $30 \%$ for thermopower was seen for thin nanowire due to hole-dominant diffusive thermoelectric generation.

The second method has addressed the shortcomings of the method mentioned above by suspending the nanowire [60,61] as seen in Fig. 6. A higher temperature gradient can be maintained since the sensing points are suspended. Thermal conductivity is calculated by considering the diameter and the conduction length of the nanowire between two suspended structures, and the difference in temperature between the two ends [38]. Because of the complexity of the measurement device and sequential fabrication steps, a nanowire is picked from the bulk sample and placed on the fabricated device. A few additional steps are required after placing the nanowire, such as focused ion beam deposition of platinum to make the electrical contacts $[60,61]$ and fixing the nanowire in its place (Fig. 6(b)). This approach provides good electrical contacts [62] which is critical to make reliable measurements. The accurate positioning of the nanowires on the suspended devices is a major challenge. One can choose a large enough nanowire visible under a microscope [60] or use a drop or two of a very dilute nanowire dispersion hoping to get by 

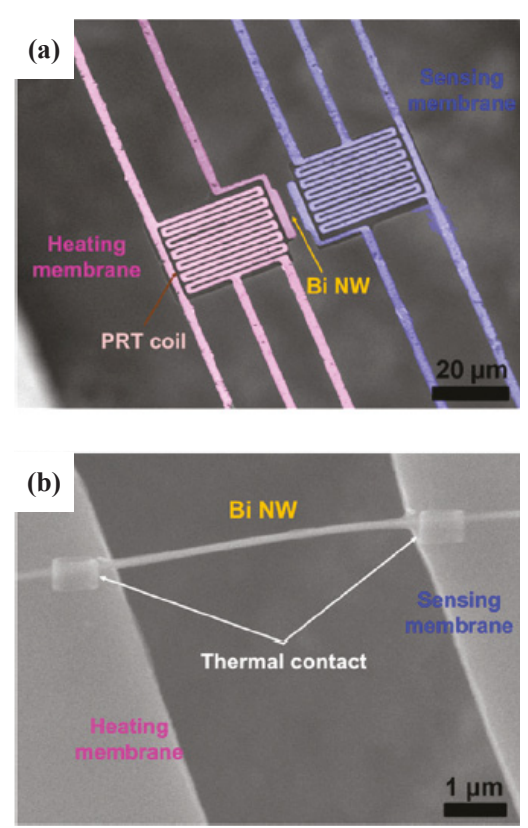

Fig. 6. (a) SEM image of the suspended microdevice for measuring the thermal conductivity of individual Bi nanowires. (b) SEM image of an individual Bi nanowire placed between the heating membrane and sensing membrane. Reproduced with permission from [44].

chance a single nanowire [63-65]. Direct growth of the nanowire on the device has also been suggested but usually ruled out due to the high temperatures involved in the growth process [61].

\section{CONCLUDING REMARKS}

The potential of nanowires to construct thermoelectric devices with higher efficiency compared to bulk materials and thin films is well recognized at present. The literature on theoretical studies and experimental attempts to make measurements has been extensive as reviewed here. But a glance at this literature and comparing different reports on the same material reveals significant disagreement between theoretical predictions and experimental measurements as well as among measurements from various groups, as documented well by Duarte [68]. For example, Ono et al. [60] reported that the thermopower of a suspended $200 \mathrm{~nm} \times 800 \mathrm{~nm} \mathrm{Bi}_{2} \mathrm{Te}_{3}$ bundle is less than that of the bulk material; however, Wang et al. [66] claimed the opposite for a parallel array of $\mathrm{Bi}_{2} \mathrm{Te}_{3}$ nanowires. Zhou et al. [65] attributed these inconsistencies to small stoichiometric differences, causing $\mathrm{S}$ to vary greatly. A significantly different thermopower with an opposite sign was also reported for bismuth nanowires with a diameter of 150-480 nm in comparison with bulk bismuth [67]. Theoretical predictions by Mingo [33] showed InSb as an excellent thermoelectric material with a large figure of merit for a nanowire with a diameter around $20 \mathrm{~nm}$, but when Zhou et al. [58] found that the thermopower of a $40 \mathrm{~nm}$ wire did not increase even relative to bulk InSb. This discrepancy was attributed to phonon effects or energy spectrum dispersion near the Fermi level. When more study was done by the same group, a thermopower an order of magnitude lower than bulk was reported [57]. While theoretical investigations show an increase in thermopower with a reduction in size, it has been seen in many cases that a reduction in size not only does not increase the thermopower but actually decreases it [68].

The discrepancies mentioned above are indicative of the im- maturity of the field at present. There are numerous challenges related to nanowire diameter control, production of nanowires with very small diameters (less than $10 \mathrm{~nm}$ ), doping of nanowires, manipulation of single nanowires for measurement purposes, reliable contacts, measurement accuracy and others. Fabrication of realistic nanowire-based products has not been reported yet and will have to await finding solutions to the above problems.

\section{ACKNOWLEDGMENTS}

This work was supported by the World Class University program through the National Research Foundation of Korea funded by the Ministry of Education, Science and Technology under Project R31-10100.

\section{REFERENCES}

[1] T. M. Tritt, Annu. Rev. Mater. Res. 41, 433 (2011) [http://dx.doi. org/10.1146/annurev-matsci-062910-100453].

[2] D. K. C. MacDonald, Thermoelectricity: An Introduction to the Principles (John Wiley \& Sons, New York, 1962)

[3] R. R. Heikes and R. W. Ure, Thermoelectricity: Science and Engineering (Interscience Publishers, New York, 1961).

[4] I. B. Cadoff and E. Miller, Thermoelectric Materials and Devices (Reinhold Publishing, New York, 1960).

[5] P. H. Egli, Thermoelectricity: Including the Proceedings of the Conference on Thermoelectricity, September, 1958 (John Wiley \& Sons, New York, 1960).

[6] L. D. Chen, X. Y. Huang, M. Zhou, X. Shi, and W. B. Zhang, J. Appl. Phys. 99, 064305 (2006) [http://dx.doi. org/10.1063/1.2180432].

[7] L. D. Zhao, B. P. Zhang, J. F. Li, M. Zhou, W. S. Liu, and J. Liu, J. Alloys Compd. 455, 259 (2008) [http://dx.doi.org/10.1016/ j.jallcom.2007.01.015].

[8] X. Y. Zhao, X. Shi, L. D. Chen, W. Q. Zhang, W. B. Zhang, and Y. Z. Pel, J. Appl. Phys. 99, 053711 (2006) [http://dx.doi. org/10.1063/1.2172705].

[9] J. R. Sootsman, H. Kong, C. Uher, J. J. D'Angelo, C.-I. Wu, T. P. Hogan, T. Caillat, and M. G. Kanatzidis, Angew. Chem. Int. Ed. 47, 8618 (2008) [http://dx.doi.org/10.1002/anie.200803934].

[10] H. Li, X. Tang, X. Su, and Q. Zhang, Appl. Phys. Lett. 92, 202114 (2008) [http://dx.doi.org/10.1063/1.2936277].

[11] W. Kim, J. Zide, A. Gossard, D. Klenov, S. Stemmer, A. Shakouri, and A. Majumdar, Phys. Rev. Lett. 96, 045901 (2006) [http:// dx.doi.org/10.1103/PhysRevLett.96.045901].

[12] A. Majumdar, Science 303, 777 (2004) [http://dx.doi. org/10.1126/science.1093164].

[13] J. S. Rhyee, K. H. Lee, S. M. Lee, E. Cho, S. I. Kim, E. Lee, Y. S. Kwon, J. H. Shim, and G. Kotliar, Nature 459, 965 (2009) [http:// dx.doi.org/10.1038/nature08088].

[14] A. F. Ioffe, Semiconductor Thermoelements, and Thermoelectric Cooling (Infosearch, London, UK, 1957).

[15] Jet Propulsion Laboratory. Voyager. Retrieved November, 2011 from http://voyager.jpl.nasa.gov.

[16] The Auto Channel. Retrieved November, 2011 from http://www. theautochannel.com.

[17] Hui Mao. Retrieved November, 2011 from http://www.huimao. $\mathrm{com} /$.

[18] TES NewEnergy Corporation. Retrieved November, 2011 from http://www.tes-ne.com/.

[19] M. S. Dresselhaus, G. Chen, M. Y. Tang, R. Yang, H. Lee, D. Wang, Z. Ren, J. P. Fleurial, and P. Gogna, Adv. Mater. 19, 1043 (2007) [http://dx.doi.org/10.1002/adma.200600527]. 
[20] M. G. Kanatzidis, Chem. Mater. 22, 648 (2010) [http://dx.doi. org/10.1021/cm902195j].

[21] A. Boukai, K. Xu and J. Heath, Adv. Mater. 18, 864, (2006) [http:// dx/doi.org/10.1002/adma200502194].

[22] T. E. Humphrey and H. Linke, Phys. Rev. Lett. 94, 096601 (2005) [http://dx.doi.org/10.1103/PhysRevLett.94.096601].

[23] L. D. Hicks and M. S. Dresselhaus, Phys. Rev. B 47, 12727 (1993) [http://dx.doi.org/10.1103/PhysRevB.47.12727].

[24] H. Ohta, S. Kim, Y. Mune, T. Mizoguchi, K. Nomura, S. Ohta, T. Nomura, Y. Nakanishi, Y. Ikuhara, M. Hirano, H. Hosono, and K. Koumoto, Nat. Mater. 6, 129 (2007) [http://dx.doi.org/10.1038/ nmat1821].

[25] J. F. Li, W. S. Liu, L. D. Zhao, and M. Zhou, NPG Asia Mater. 2, 152 (2010) [http://dx.doi.org/10.1038/asiamat.2010.138].

[26] N. Mingo, D. Hauser, N. P. Kobayashi, M. Plissonnier, and A. Shakouri, Nano Lett. 9, 711 (2009) [http://dx.doi.org/10.1021/ n18031982].

[27] R. Venkatasubramanian, E. Siivola, T. Colpitts, and B. O'Quinn, Nature 413, 597 (2001) [http://dx.doi.org/10.1038/35098012].

[28] T. C. Harman, P. J. Taylor, M. P. Walsh, and B. E. LaForge, Science 297, 2229 (2002) [http://dx.doi.org/10.1126/science.1072886].

[29] W. Liang, O. Rabin, A. I. Hochbaum, M. Fardy, M. Zhang, and P. Yang, Nano Res. 2, 394 (2009) [http://dx.doi.org/10.1007/ s12274-009-9039-2].

[30] L. D. Hicks and M. S. Dresselhaus, Phys. Rev. B 47, 16631 (1993) [http://dx.doi.org/10.1103/PhysRevB.47.16631].

[31] A. Casian, I. Sur, A. Sandu, H. Scherrer, and S. Scherrer, Proceedings of the 16th International Conference on Thermoelectrics (Dresden, German 1997 Aug. 26-29, IEEE) p. 442. [http:// dx.doi.org/10.1109/ICT.1997.667182].

[32] M. S. Dresselhaus, G. Dresselhaus, X. Sun, Z. Zhang, S. B. Cronin, and T. Koga, Phys. Solid State 41, 679 (1999) [http://dx.doi. org/10.1134/1.1130849].

[33] N. Mingo, Appl. Phys. Lett. 85, 5986 (2004) [http://dx.doi. org/10.1063/1.1829391].

[34] N. Mingo, Appl. Phys. Lett. 84, 2652 (2004) [http://dx.doi. org/10.1063/1.1695629].

[35] T. T. M. Vo, A. J. Williamson, V. Lordi, and G. Galli, Nano Lett. 8, 1111 (2008) [http://dx.doi.org/10.1021/nl073231d].

[36] A. I. Boukai, Y. Bunimovich, J. Tahir-Kheli, J. K. Yu, W. A. Goddard Iii, and J. R. Heath, Nature 451, 168 (2008) [http://dx.doi. org/10.1038/nature06458].

[37] S. H. Lee, W. Shim, S. Y. Jang, J. W. Roh, P. Kim, J. Park, and W. Lee, Nanotechnology 22, 295707 (2011) [http://dx.doi. org/10.1088/0957-4484/22/29/295707].

[38] M. Fardy, A. L. Hochbaum, J. Goldberger, M. M. Zhang, and P. Yang, Adv. Mater. 19, 3047 (2007) [http://dx.doi.org/10.1002/ adma.200602674].

[39] G. Zhang, Q. Yu, W. Wang, and X. Li, Adv. Mater. 22, 1959 (2010) [http://dx.doi.org/10.1002/adma.200903812].

[40] G. Zhang, W. Wang, and X. Li, Adv. Mater. 20, 3654 (2008) [http:// dx.doi.org/10.1002/adma.200800162].

[41] J. Kang, J. W. Roh, W. Shim, J. Ham, J. S. Noh, and W. Lee, Adv. Mater. 23, 3414 (2011) [http://dx.doi.org/10.1002/ adma.201101460].

[42] M. Hu, K. P. Giapis, J. V. Goicochea, X. Zhang, and D. Poulikakos, Nano Lett. 11, 618 (2011) [http://dx.doi.org/10.1021/ nl103718a].

[43] A. I. Hochbaum, R. Chen, R. D. Delgado, W. Liang, E. C. Garnett, M. Najarian, A. Majumdar, and P. Yang, Nature 451, 163 (2008) [http://dx.doi.org/10.1038/nature06381].

[44] J. W. Roh, K. Hippalgaonkar, J. H. Ham, R. Chen, M. Z. Li, P. Ercius, A. Majumdar, W. Kim, and W. Lee, ACS Nano 5, 3954 (2011) [http://dx.doi.org/10.1021/nn200474d].

[45] A. L. Moore, M. T. Pettes, F. Zhou, and L. Shi, J. Appl. Phys. 106,
034310 (2009) [http://dx.doi.org/10.1063/1.3191657].

[46] M. Meyyappan and M. K. Sunkara, Inorganic Nanowires: Applications, Properties, and Characterization (CRC Press, Boca Raton, 2010).

[47] K. Devami, D. Kang, J. Lee, and M. Meyyappan, Chem. Phys. Lett., 504, 62 (2011) [http://dx.doi.org/10.1016/ j.cplett.2011.01.053].

[48] K. Davami, H. M. Ghassemi, X. Sun, R. S. Yassar, J. S. Lee, and M. Meyyappan, Nanotechnology 22, 435204 (2011) [http://dx.doi. org/10.1088/0957-4484/22/43/435204].

[49] M. Tian, J. Wang, J. Kurtz, T. E. Mallouk, and M. H. W. Chan, Nano Lett. 3, 919 (2003) [http://dx.doi.org/10.1021/nl034217d].

[50] F. S. S. Chien, C. L. Wu, Y. C. Chou, T. T. Chen, S. Gwo, and W. F. Hsieh, Appl. Phys. Lett. 75, 2429 (1999) [http://dx.doi. org/10.1063/1.125037].

[51] Y. J. Chen, J. H. Hsu, and H. N. Lin, Nanotechnology 16, 1112 (2005) [http://dx.doi.org/10.1088/0957-4484/16/8/020].

[52] S. A. Harfenist, S. D. Cambron, E. W. Nelson, S. M. Berry, A. W. Isham, M. M. Crain, K. M. Walsh, R. S. Keynton, and R. W. Cohn, Nano Lett. 4, 1931 (2004) [http://dx.doi.org/10.1021/ nl048919u]

[53] F. Zhou, A. L. Moore, M. T. Pettes, Y. Lee, J. H. Seol, Q. L. Ye, L. Rabenberg, and L. Shi, J. Phys. D: Appl. Phys. 43, 025406 (2010) [http://dx.doi.org/10.1088/0022-3727/43/2/025406].

[54] Y. H. Tang, Y. F. Zheng, C. S. Lee, and S. T. Lee, Chem. Phys. Lett. 328, 346 (2000) [http://dx.doi.org/10.1016/S00092614(00)00862-9].

[55] A. R. Abramson, W. C. Kim, S. T. Huxtable, H. Yan, Y. Wu, A. Majumdar, C. L. Tien, and P. Yang, J. Microelectromech. Syst. 13, 505 (2004) [http://dx.doi.org/10.1109/jmems.2004.828742].

[56] G. U. Sumanasekera, L. Grigorian, and P. C. Eklund, Meas. Sci. Technol. 11, 273 (2000) [http://dx.doi.org/10.1088/09570233/11/3/315].

[57] J. H. Seol, A. L. Moore, S. K. Saha, F. Zhou, L. Shi, Q. L. Ye, R. Scheffler, N. Mingo, and T. Yamada, J. Appl. Phys. 101, 023706 (2007) [http://dx.doi.org/10.1063/1.2430508].

[58] F. Zhou, J. H. Seol, A. L. Moore, L. Shi, Q. L. Ye, and R. Scheffler, J. Phys. Condens. Matter 18, 9651 (2006) [http://dx.doi. org/10.1088/0953-8984/18/42/011].

[59] Y. M. Zuev, J. S. Lee, C. Galloy, H. Park, and P. Kim, Nano Lett. 10, 3037 (2010) [http://dx.doi.org/10.1021/nl101505q].

[60] T. Ono, C. C. Fan, and M. Esashi, J. Microelectromech. Syst. 15, 1 (2005) [http://dx.doi.org/10.1088/0960-1317/15/1/001].

[61] L. Shi, D. Li, C. Yu, W. Jang, D. Kim, Z. Yao, P. Kim, and A. Majumdar, J. Heat Transfer 125, 881 (2003) [http://dx.doi. org/10.1115/1.1597619].

[62] H. E. Romero, G. U. Sumanasekera, G. D. Mahan, and P. C. Eklund, Phys. Rev. B 65, 205410 (2002) [http://dx.doi. org/10.1103/PhysRevB.65.205410].

[63] L. Shi, Q. Hao, C. Yu, N. Mingo, X. Kong, and Z. L. Wang, Appl. Phys. Lett. 84, 2638 (2004) [http://dx.doi. org/10.1063/1.1697622].

[64] F. Zhou, J. Szczech, M. T. Pettes, A. L. Moore, S. Jin, and L. Shi, Nano Lett. 7, 1649 (2007) [http://dx.doi.org/10.1021/ nl0706143].

[65] J. Zhou, C. Jin, J. H. Seol, X. Li, and L. Shi, Appl. Phys. Lett. 87, 133109 (2005) [http://dx.doi.org/10.1063/1.2058217].

[66] W. Wang, F. Jia, Q. Huang, and J. Zhang, Microelectron. Eng. 77, 223 (2005) [http://dx.doi.org/10.1016/j.mee.2004.11.005].

[67] A. Nikolaeva, T. E. Huber, D. Gitsu, and L. Konopko, Phys. Rev. B 77, 035422 (2008) [http://dx.doi.org/10.1103/PhysRevB.77.035422].

[68] N. B. Duarte, Thermopower measurement of goild nanowire systems using a micromachined workbench, PhD dissertation (The Pennsylvania State University, State College, PA, 2008) 